\title{
Changes in Blood Volume and Colloid Osmotic Pressure during Fluid Absorption in Patients Undergoing Endoscopic Urosurgery: An Observational Study ${ }^{*}$
}

\author{
Kaori Yagi $^{1}$, Chihiro Kamagata ${ }^{1}$, Masashi Ishikawa ${ }^{1}$, Yukihiro Kondo ${ }^{2}$, Atsuhiro Sakamoto ${ }^{1}$ \\ ${ }^{1}$ Department of Anesthesiology and Pain Medicine, Graduate School of Medicine, Nippon Medical School, Tokyo, Japan; ${ }^{2}$ Depart- \\ ment of Urology, Nippon Medical School, Tokyo, Japan. \\ Email: fuku-kaori@nms.ac.jp
}

Received September $8^{\text {th }}, 2013$; revised October $1^{\text {st }}, 2013$; accepted October $17^{\text {th }}, 2013$

Copyright (C) 2013 Kaori Yagi et al. This is an open access article distributed under the Creative Commons Attribution License, which permits unrestricted use, distribution, and reproduction in any medium, provided the original work is properly cited.

\begin{abstract}
Background and Objective: Anesthesiologists need to be familiar with perioperative changes in blood volume (BV); however, there is no standard method for repeated evaluation of BV over a short interval of time. We evaluated BV in the operation room using repeatable estimation methods. Method: Eighty-five ASA physical status I-II patients scheduled to undergo endoscopic urosurgery using irrigation fluid under general anesthesia at Nippon Medical School Hospital were included in this study. Irrigation with $3 \%$ sorbitol in water was commenced after establishment of general anesthesia and volumetric fluid balance, which was defined as control water balance (WB). Hematocrit (Hct), colloid osmotic pressure (COP), total protein (TP) and albumin (Alb) were repeatedly determined before and during anesthesia. BV was calculated using Allen's formula and the changes in Hct, COP, TP and Alb. Main Outcome Measures: The main outcome was the accuracy of measuring changes in $\mathrm{BV}(\triangle \mathrm{BV})$ calculated using the four serum markers. WB and the estimated $\triangle \mathrm{BV}$ calculated from Hct, COP, TP and Alb ( $\triangle \mathrm{BV}$-Hct, $\triangle \mathrm{BV}-\mathrm{COP}, \Delta \mathrm{BV}-\mathrm{TP}$, and $\Delta \mathrm{BV}$-Alb) were analysed using Pearson's correlation coefficient test and Bland-Altman analysis. Results: Sixty-five patients were excluded. In the remaining 20 patients, there was a significant correlation between $\mathrm{WB}$ and $\Delta \mathrm{BV}-\mathrm{COP}\left(\mathrm{R}^{2}=0.72 ; \mathrm{P}<0.01\right), \mathrm{WB}$ and $\triangle \mathrm{BV}-\mathrm{TP}\left(\mathrm{R}^{2}=0.59 ; \mathrm{P}<0.01\right)$ and WB and $\triangle \mathrm{BV}-\mathrm{Alb}\left(\mathrm{R}^{2}=0.57 ; \mathrm{P}<0.01\right)$, while there was no correlation between $\mathrm{WB}$ and $\Delta \mathrm{BV}$-Hct $\left(\mathrm{R}^{2}=0.06\right)$. Conclusion: $\triangle \mathrm{BV}-\mathrm{COP}, \triangle \mathrm{BV}-\mathrm{TP}$ and $\triangle \mathrm{BV}$-Alb had correlation with $\mathrm{WB}$. However, since COP can be measured repeatedly with simplified instruments under selected clinical circumstances, while TP and Alb cannot. $\mathrm{COP}$ is the most useful marker to measure $\triangle \mathrm{BV}$ during perioperative period. Hct does not allow precise estimation of $\triangle \mathrm{BV}$.
\end{abstract}

Keywords: Colloid Osmotic Pressure; Blood Volume; Perioperative Period

\section{Introduction}

Measurement of blood volume (BV) is a perioperative concern for the anesthetist. The standard method for measuring $\mathrm{BV}$ directly is the marker dilution technique, using a radioisotope $[1,2]$. However, it is difficult to use this method for serial measurements of BV over a short interval of time because radioisotopes must be used in a special radiation-permitted area, and these tracers are retained in the blood for days. Yet, it is necessary for us to be able to measure BV safely, repeatedly, accurately and conveniently. Therefore, we questioned whether a substance that naturally occurs in the intravascular

${ }^{*}$ Conflicts of interest: None declared. space could be used as an indicator of plasma dilution, allowing safe and repeated measurements without administration of a dilution drug. Previously, changes in hematocrit (Hct), total protein (TP) and plasma colloid osmotic pressure (COP) have been used to estimate changes in $\mathrm{BV}$ in patients undergoing minor surgery under general anesthesia [3]. Although these markers can be measured safely, repeatedly, accurately and conveniently, it was not clear which of these markers are accurate representatives of actual BV changes. The purpose of this study was to evaluate the accuracy of measuring changes in $\mathrm{BV}$ using Hct, $\mathrm{COP}, \mathrm{TP}$, and albumin (Alb) as dilution markers, to determine the most useful marker during the perioperative period. 


\section{Patients and Methods}

The study protocol was registered with the UMIN Clinical Trials Registry (Trial No. UMIN000007560). Ethical approval for this study (Protocol number 223009) was provided by the Institutional Review Board of Nippon Medical School Hospital, Tokyo, Japan, (Chairperson: Hospital director Y Fukunaga) on 30 November 2011. We obtained written informed consent from all patients the day before surgery. Eighty-five male patients undergoing transurethral resection of bladder tumors (TUR-Bt) and transurethral resection of the prostate (TUR-P) at the Nippon Medical School Hospital between April 2012 and March 2013 were included in this study. All patients were American Society of Anesthesiologists (ASA) physical status I-II, with no preexisting renal, hepatic or severe heart disease.

All patients fasted from $9 \mathrm{pm}$ on the eve of surgery, and no intravenous fluids were administered before the induction of anesthesia. On arrival at the operating room, a 22-G cannula was placed in the left radial artery under local anesthesia with $1 \%$ lidocaine, for collection of blood samples during the study period. Before anesthesia, Hct, TP, Alb and COP were measured. These parameters were also measured $30 \mathrm{~min}$ after the induction of anesthesia, at which time irrigation was started (Anesth30), and then at 30-min intervals thereafter during anesthesia (i.e., Anesth-60, Anesth-90 etc.). We also collected blood samples at the end of the surgical procedure ("Anesth-end") and $30 \mathrm{~min}$ after tracheal extubation ("Recovery"). A flow chart of the timing of blood sampling following anesthesia and irrigation is shown in Figure 1.

After baseline measurements, a peripheral intravenous cannula (20-G) was inserted to administer normal saline throughout the study period, at a rate of $1 \mathrm{~mL} \cdot \mathrm{kg}^{-1} \cdot \mathrm{h}^{-1}$, using an infusion pump. Hct, TP and Alb were measured using standard techniques. COP was measured with a colloid osmometer (Colloid-4420, Wescor Inc., Logan, UT, USA). The colloid osmometer employs a method referred to as transudation, which involves passing water molecules and diffusible solute particles through a synthetic semi-permeable membrane that has a diffusion cut-off of 30,000 Da [4]. The membrane separates the

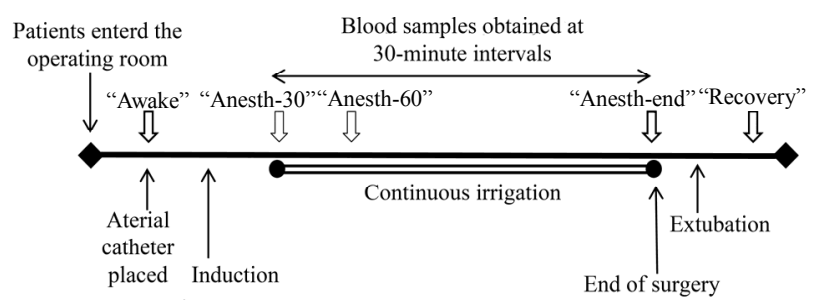

Figure 1. Schematic diagram of the study protocol. specimen solution from a reference solution after the samples are injected into the reference chamber. The fluid then moves through the membrane and into the sample chamber until the hydrostatic pressure reaches equilibrium. This pressure is measured by a piezoelectric pressure transducer.

General anesthesia was induced with propofol (2.0 $\left.\mathrm{mg} \cdot \mathrm{kg}^{-1}\right)$ and rocuronium $\left(0.6 \mathrm{mg} \cdot \mathrm{kg}^{-1}\right)$. Endotracheal intubation was performed about $5 \mathrm{~min}$ after induction. Patients were mechanically ventilated with oxygen (2 $\left.\mathrm{L} \cdot \mathrm{min}^{-1}\right)$ and air $\left(4 \mathrm{~L} \cdot \mathrm{min}^{-1}\right)$ at a tidal volume of 8 $\mathrm{mL} \cdot \mathrm{kg}^{-1}$ and respiratory rate of $8-12$ breaths $\cdot \mathrm{min}^{-1}$, to maintain an end-tidal $\mathrm{CO}_{2}$ of $35-40 \mathrm{mmHg}$ during the anesthetic period. Anesthesia was maintained with sevoflurane supplemented with remifentanil $\left(0.1 \mu \mathrm{g} \cdot \mathrm{kg}^{-1} \cdot \mathrm{min}^{-1}\right)$. The sevoflurane concentration was controlled to maintain SBP within $80 \%-120 \%$ of awake values. If hypotension occurred, it was treated with $4 \mathrm{mg}$ ephedrine intravenously. At the end of surgery, sevoflurane and remifentanil were discontinued, and sugammadex $\left(4 \mathrm{mg} \cdot \mathrm{kg}^{-1}\right)$ was injected to reverse the effects of rocuronium. Postoperatively, the patients were left undisturbed while breathing supplemental oxygen $\left(6 \mathrm{~L} \cdot \mathrm{min}^{-1}\right)$ administered via a face mask.

The BV of each patient was calculated using the formula of Allen and colleagues $\left(\mathrm{BV}_{\text {Allen }}\right)$ [5], which indicates the $\mathrm{BV}$ in the awake condition $[6,7]$.

Equation for males: $\mathrm{BV}_{\text {Allen }}=0.417 \times$ height $^{3}(\mathrm{~m})+$ $0.045 \times$ body weight $(\mathrm{kg})-0.03(\mathrm{~L})$

$\mathrm{BV}$ at each sampling time was estimated from the baseline $\mathrm{BV}_{\text {Allen }}$ and the subsequent changes in Hct, COP, $\mathrm{TP}$ and Alb respectively. For example, BV estimated from the change in COP (BV-COP) at "Anesth-30" was calculated by the following formula:

BV-COP $\left(\right.$ Anesth-30) $=\mathrm{BV}_{\text {Allen }}($ Awake $) \times[\mathrm{COP}$ (Awake)/COP (Anesth-30)]

The change in $\mathrm{BV}(\Delta \mathrm{BV})$ calculated with each serum marker was defined as the difference between the baseline BV (Anaesth-30) and the BV at the end of irrigation (Anesth-end). Blood loss was calculated by assaying Hct and the volume of the discarded irrigation fluid, using the formula:

Blood Loss $(\mathrm{BL})=\mathrm{BV} \times$ Hct $($ of the discarded irrigation fluid)/Hct (Recovery)

WB was calculated by the following formula:

$\mathrm{WB}=$ Irrigation $($ Used $)-$ Irrigation $($ Discarded $)+\mathrm{BL}$ where, Irrigation (Used) is the amount of irrigation fluid used and Irrigation (Discarded) is the amount of fluid discarded. These fluid volumes were exactly measured using a graduated cylinder (5 L). The fluid used for irrigation was 3\% sorbitol in water (Baxter Ltd., Deerfield, IL, USA), with an osmolarity of about 165 $\mathrm{mOsm} \cdot \mathrm{L}^{-1}$. The irrigation fluid bag was placed at a 
height of $50 \mathrm{~cm}$ above the bladder.

All data are expressed as mean \pm standard deviation (SD) unless otherwise stated. $\mathrm{P}<0.05$ was considered to be significant. The correlations between $\mathrm{WB}$ and $\triangle \mathrm{BV}$ calculated from Hct, COP, TP and Alb $(\triangle \mathrm{BV}-\mathrm{Hct}, \mathrm{BV}-$ $\mathrm{COP}, \mathrm{V}-\mathrm{TP}$ and $\Delta \mathrm{BV}-\mathrm{Alb}$, respectively) were analyzed by pearson's correlation coefficient test. When a significant correlation was found, the agreement between WB and $\Delta \mathrm{BV}$ was assessed by Bland-Altman analysis.

\section{Results}

A total of eighty-five patients were recruited for the study and operated upon. Table 1 summarizes the clinical characteristics of the eighty-five patients included in the study. Of them, we excluded three patients who had a high amount of absorption, resulting in hyponatremia, as they had a large amount of bleeding and required transfusion or colloid administration. The correlations between $\mathrm{WB}$ and $\Delta \mathrm{BV}$-Hct, $\Delta \mathrm{BV}-\mathrm{COP}, \Delta \mathrm{BV}-\mathrm{TP}$ and $\Delta \mathrm{BV}-\mathrm{Alb}$ for the remaining eighty-two patients are shown in Figure 2. However, as most data showed that WB was close to zero, indicating that patients had little bleeding and absorption, patients with WB less than $300 \mathrm{ml}$ were excluded from the analysis (Excluded area: Dot boxes in Figure 2).

As a result, 12 patients undergoing TUR-P and 8 patients undergoing TUR-Bt were selected for further study. Table 2 summarizes the clinical characteristics of the 20 patients included in the study. The correlations between $\mathrm{WB}$ and $\Delta \mathrm{BV}-\mathrm{COP}, \Delta \mathrm{BV}-\mathrm{TP}$ and $\Delta \mathrm{BV}$-Alb except $\Delta \mathrm{BV}$ Hct are shown in Figure 3. There was a significant correlation between $\mathrm{WB}$ and $\triangle \mathrm{BV}-\mathrm{COP}\left(\mathrm{R}^{2}=0.72 ; \mathrm{P}<\right.$ $0.01)$, WB and $\triangle \mathrm{BV}-\mathrm{TP}\left(\mathrm{R}^{2}=0.59 ; \mathrm{P}<0.01\right)$, and $\mathrm{WB}$ and $\Delta \mathrm{BV}-\mathrm{Alb}\left(\mathrm{R}^{2}=0.57 ; \mathrm{P}<0.01\right)$. On the other hand, there was no correlation between WB and $\triangle \mathrm{BV}$-Hct $\left(\mathrm{R}^{2}\right.$ $=0.06$ ). Bland-Altman analysis of $\mathrm{WB}$ and $\triangle \mathrm{BV}-\mathrm{COP}$, $\triangle \mathrm{BV}-\mathrm{TP}$ and $\triangle \mathrm{BV}$-Alb are shown in Figure 4. BlandAltman analysis of WB and $\triangle \mathrm{BV}-\mathrm{COP}$ indicated a bias of 0.04 with limits of agreement from -0.14 to 0.22 , with agreement in 18 of the 20 pairs of samples $(90 \%)$. BlandAltman analysis of WB and $\triangle \mathrm{BV}$-TP indicated a bias of 0.12 with limits of agreement from -0.10 to 0.35 , with agreement in 17 of the 20 pairs of samples (85\%). BlandAltman analysis of WB and $\triangle \mathrm{BV}$-Alb indicated a bias of 0.08 with limits of agreement from -0.08 to 0.25 , with agreement in 16 of the 20 pairs of samples $(80 \%)$.

Table 1. Data and clinical characteristics of study subjects $(\mathbf{n}=\mathbf{8 5})$.

\begin{tabular}{cc}
\hline Number of patients & $\mathrm{n}=85$ \\
\hline Age (yr) & $68.9 \pm 11.2(33-89)$ \\
Height (cm) & $163.4 \pm 7.71(152-180)$ \\
Weight (kg) & $61.7 \pm 9.96(44-90)$ \\
Time of operation (min) & $101 \pm 54.1(16-280)$ \\
Blood loss (ml) & $43.1 \pm 67.1(0-2146)$ \\
Irrigation fluid (L) & $16.0 \pm 1.38(1.50-57.0)$ \\
Discarded fluid (L) & $15.4 \pm 1.34(1.50-56.2)$ \\
Water Balance (L) & $0.35 \pm 0.88(-0.1-1.25)$ \\
Weight of the tumor (g) & $9.61 \pm 17.4(0-55)$ \\
BVAllen in the "Awake" state (L) & $4.9 \pm 0.54(2.91-5.86)$ \\
\hline
\end{tabular}

Table 2. Data and clinical characteristics of study subjects (n= 20).

\begin{tabular}{cc}
\hline Number of patients & $\mathrm{n}=20$ \\
\hline Age (yr) & $69.0 \pm 12.8(44-87)$ \\
Height (cm) & $167.8 \pm 5.1(158-176)$ \\
Weight (kg) & $64.5 \pm 10.2(48-86)$ \\
Time of operation (min) & $148 \pm 64.7(60-280)$ \\
Blood loss (ml) & $156.0 \pm 46(60-446)$ \\
Irrigation fluid (L) & $29.7 \pm 1.93(10.5-57.0)$ \\
Discarded fluid (L) & $29.2 \pm 19.2(10.0-56.2)$ \\
Water Balance (L) & $0.51 \pm 0.20(0.3-1.25)$ \\
Weight of tumor (L) & $14.9 \pm 14.5(1-55)$ \\
BVAllen in the “Awake" state (L) & $4.74 \pm 0.40(3.56-5.86)$ \\
\hline
\end{tabular}
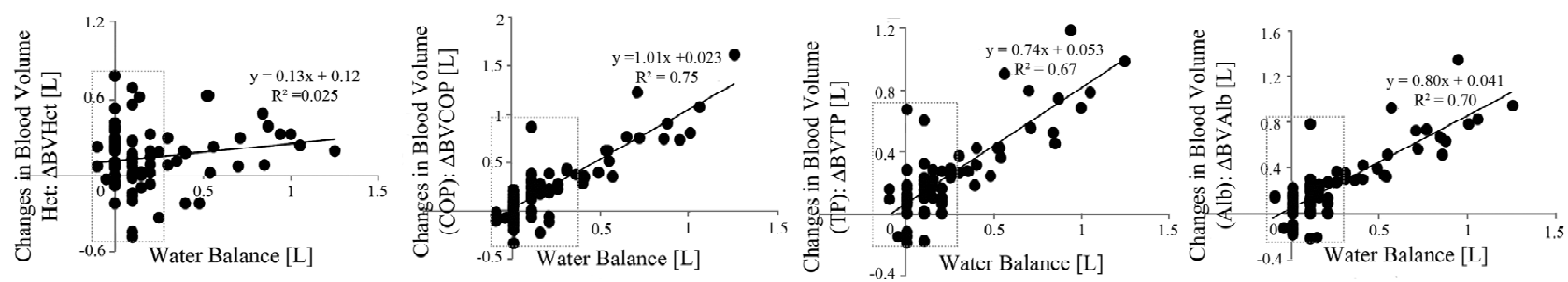

Figure 2. Relationships between changes in blood volume during irrigation, calculated from hematocrit $(\Delta \mathrm{BV}-\mathrm{Hct})$, colloid osmotic pressure $(\triangle B V-C O P)$, total protein $(\triangle B V-T P)$ and albumin $(\triangle B V-A l b)$, and water balance $(W B)(n=82)$. The areas enclosed in the dot boxes are excluded in Figure 3. 

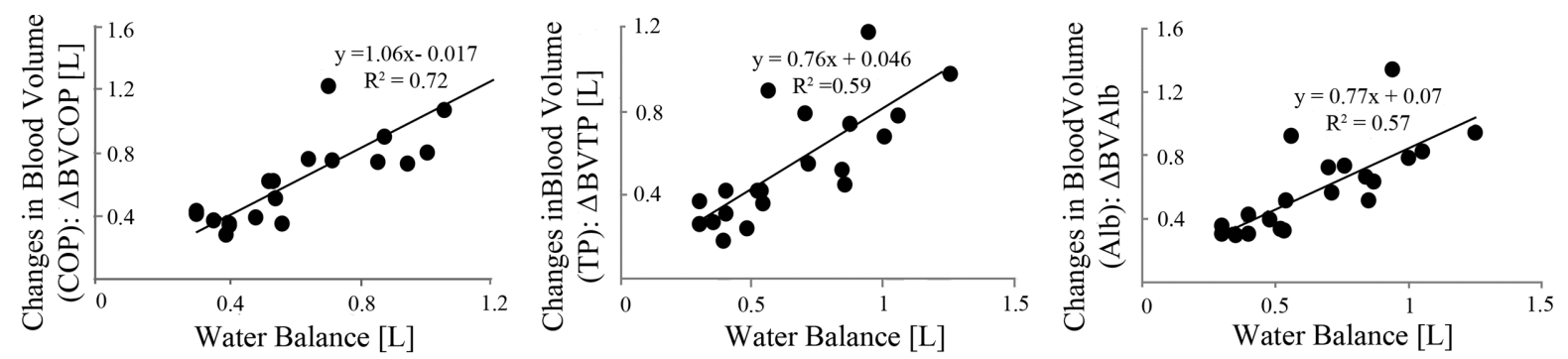

Figure 3. Relationships between changes in blood volume during irrigation, calculated from colloid osmotic pressure ( $\triangle \mathrm{BV}$ COP $)$, total protein $(\triangle B V-T P)$ and albumin $(\triangle B V-A l b)$, and water balance $(W B)(n=20)$.
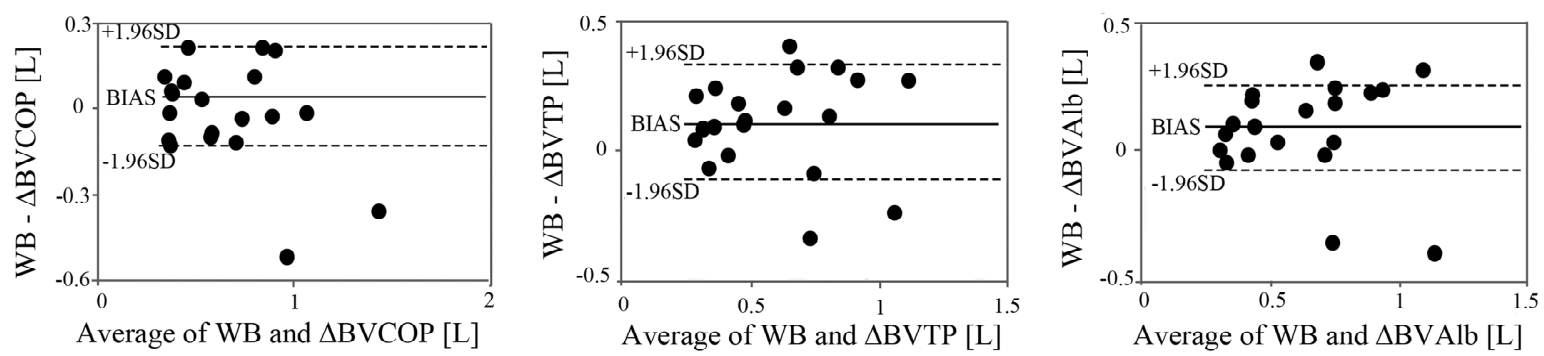

Figure 4. Bland-Altman bias plot comparing water balance (WB) and changes in blood volume calculated from colloid osmotic pressure $(\triangle B V-C O P)$, total protein $(\triangle B V-T P)$ and albumin $(\triangle B V-A l b)$. The fine lines indicate the $95 \%$ confidence interval (CI) and show the limits of agreement, which was 1.96 standard deviation (SD) around the mean difference (bias; heavy line) $(\mathbf{n}=\mathbf{2 0})$.

There was an additional margin of error in BlandAltman analysis of WB and $\triangle \mathrm{BV}-\mathrm{TP}$, and $\mathrm{WB}$ and $\triangle \mathrm{BV}$ $\mathrm{Alb}$, which indicates that $\mathrm{WB}$ is usually bigger than $\Delta \mathrm{BV}$-TP and $\Delta \mathrm{BV}$-Alb.

\section{Discussion}

Determining $\triangle \mathrm{BV}$ is an essential component of perioperative fluid management. We used Hct, COP, TP and Alb as serum markers to estimate plasma dilution, and verified the accuracy of these parameters in estimating $\triangle \mathrm{BV}$. Given the nature of these surgical procedures, transpiration probably contributed minimally to $\Delta \mathrm{BV}$. The volume of fluid infused intravenously was too small to account for the increase in BV observed during irrigation. All measurements, except those performed in the "Awake" and "Recovery" state, were performed during periods of stable anesthesia and hemodynamics. Therefore, the variation in serum markers from "Anesth-30" to "Anesth-end" was most likely due to absorption of the irrigation fluid. Measurement of changes in Hct, COP, $\mathrm{TP}$ and Alb thus appears to be a reliable method for estimating $\triangle \mathrm{BV}$ resulting from dilution or concentration of the blood during the perioperative period. In this study, we evaluated the accuracy of measuring $\Delta \mathrm{BV}$ using four markers and researched the most convenient marker among them.

A major finding of the present study was that Hct changed minimally, and $\triangle \mathrm{BV}-\mathrm{COP}, \triangle \mathrm{BV}-\mathrm{TP}$ and $\Delta \mathrm{BV}$ -
Alb have similar correlation with WB; however, $\Delta \mathrm{BV}$ $\mathrm{TP}$ and $\Delta \mathrm{BV}$-Alb underestimated WB. We propose several possible underlying mechanisms to explain these results.

First, Hct is commonly used for the long-term management of patients with chronic diseases and as a marker of dilution in the therapy of dehydration. However, there are disputes regarding the validity of using Hct for estimating the state of hydration [8,9]. Some investigators have shown that there is a difference between the anatomic width of the microvasculature and the dimensions of the space available for circulating erythrocytes. The former is termed as the "endothelial surface layer" (ESL) and the fluid in this area is without erythrocytes and is either immobile or moves very slowly. However, the volume of the ESL probably does not remain constant when plasma is replaced or diluted with artificial fluids. In such cases, the ESL can dissolve in the flowing blood, thereby decreasing the thickness of the ESL and widening the circulating column. This decreases flow resistance and increases microvessel Hct $[10,11]$. Therefore, Hct is easily changeable under clinical conditions. From this viewpoint, Hct is not a reliable dilution marker for precisely estimating minute changes in $\mathrm{BV}$.

On the other hand, plasma proteins are thought to be included in the ESL $[12,13]$. Thus, TP and Alb remain unaltered by the changing ESL. In this study, $\triangle \mathrm{BV}-\mathrm{TP}$ and $\triangle \mathrm{BV}$-Alb correlated with $\mathrm{WB}$ and were constantly 
smaller than WB with an additional margin of error in Bland-Altman analysis. This is because the hypo-osmolar sorbitol solution that was used for irrigation probably passed from the intravascular to the extravascular compartment. These results indicate that the absorbed irrigation fluid passed through the pores into the intracellular compartment at a relatively constant rate.

Finally, COP had the greatest correlation with WB. Considering the leak of absorbed irrigation fluid from the intravascular to the extravascular space, $\triangle \mathrm{BV}$-COP should have been smaller than WB. However, it did not have an additional margin of error in Bland-Altman analysis. Thus, $\triangle \mathrm{BV}-\mathrm{COP}$ was bigger than the "true" $\mathrm{BV}$ changes, indicating that $\triangle \mathrm{BV}-\mathrm{COP}$ may overestimate $\mathrm{WB}$.

COP is determined not only by albumin, but also by several other kinds of oncotic substances, including spherical colloids such as glycogen and hydroxyethyl starch, and linear colloids such as fibrinogen and dextran. As these colloid molecules are too large to pass through the pores, they do not usually leak out from the intravascular to the extravascular space, except in cases of bleeding or worsening of the patient's general condition, as with shock. The mechanism of the overestimation in $\triangle \mathrm{BV}$-COP has not been clarified. However, the fact that only $\triangle \mathrm{BV}$ COP was overestimated implies the presence of large vascular pores, restricting the passage of TP and Alb, and permitting the passage of other oncotic substances during this study. The passage of molecules across pores is more dependent on their diameter than the weight-average molecular weight (MWw) [14]. Oncotic substances with a relatively small radius can easily pass from the intravascular to the extravascular compartment, leading to a decrease in the concentration of circulating molecules and resulting in overestimation of BV. If the vascular pore is extended, there is a possibility that oncotic substances may leak out from the intravascular to the extravascular space as well.

The endothelial glycocalyx, which normally binds to large anionic molecules and prevents their extravasation, is so sensitive that an increase in capillary hydrostatic pressure caused by volume expansion can result in leakage of larger molecules from the intravascular to the extravascular space [15]. Since the induction of anesthesia causes a rapid decrease in arterial and capillary pressures, it creates a mismatch between BV and the intravascular space, which could result in some kind of endothelial change. In this way, if an appreciable number of macromolecules smaller than TP and Alb, which determine the COP, leak out of the intravascular space for some reason, the resultant lower COP may be interpreted as an increase in BV. Although we cannot explain how endoscopic urologic surgeries and general anesthesia alter microvascular permeability, capillary leak may have occurred during the procedure, explaining our results.

In this study, COP was measured at the patient's bedside using an osmometer, which is a very useful device of portable size $(3.2 \mathrm{~kg}$ ) that can measure COP in 180 300 seconds. Using this device, we could recognize rapid decreases in COP in three patients and were able to address hyponatremia at an early phase. Overestimation helped us identify even small changes with greater sensitivity. On the other hand, measurements of TP and Alb required an automated analyzer (BioMajesty JCA-BM6050; JEOL, Tokyo, Japan); this analyzer is usually set up in a large laboratory and cannot be used for simple measurements in the operating room. Hence, measuring $\triangle \mathrm{BV}-\mathrm{COP}$ may be a more convenient method of estimating WB.

Our study has several limitations. The first limitation is that we did not control for the influence of anesthesia. Irrigation was initiated $30 \mathrm{~min}$ after the induction of anesthesia, at which time there was little change in blood pressure and heart rate. Furthermore, there were minimal changes in vital signs during irrigation, indicating the absence of changing anesthetic depth. Hence, we estimated that the influence of anesthesia on the measured variables was minimal. However, the influence of anesthesia would have a little effect on the overestimation of $\triangle \mathrm{BVCOP}$.

A second limitation is that many patients had little bleeding and absorption. In the literature, the volume of blood loss (BL) during endoscopic urologic surgery of the prostate is estimated to be an average of $693 \mathrm{ml}(60$ to $2554 \mathrm{ml}$ ) [16], which is a large amount of absorption. In contrast, in our study, the volume of BL and WB were much smaller than those in this previous study. As seen in Table 2, the average BL in our study was $156 \mathrm{ml}(60$ $446 \mathrm{ml})$. Further study is therefore necessary to correlate large changes in WB with $\triangle \mathrm{BV}$.

In conclusion, although $\mathrm{COP}$ overestimates $\Delta \mathrm{BV}$, it is a useful parameter, which allows quick and easy estimation of WB, especially in situations where there is a sudden deterioration in the patient's condition. We also found that although TP and Alb can accurately estimate $\mathrm{BV}$ than COP, they are not suitable for intraoperative serial measurement. Meanwhile, Hct is not a reliable dilution marker for precise estimations of minute changes in BV. These results indicate that COP is the most suitable dilution marker in the perioperative period.

\section{Acknowledgments}

Assistance with the study: We would like to thank the concerned anesthesia personnel for general assistance in support of this study. We also thank Forte Science Communications (Tokyo, Japan) for assistance with the ma- 
nuscript.

\section{REFERENCES}

[1] M. R. Ujhelyi, A. W. Miller, S. Raibon, J. Corley, V. J. Robinson, J. J. Sims, T. Tonnessen, G. Burke, A. llebekk and D. L. Rutlen, "Endotoxemia Alters Splanchnic Capacitance," Shock (Augusta, Ga.), Vol. 14, No. 1, 2000, pp. 68-72.

[2] C. Veillon, K. Y. Patterson, D. A. Nagey and A. M. Tehan, "Measurement of Blood Volume with an Enriched Stable Isotope of Chromium (53Cr) and Isotope Dilution by Combined Gas Chromatography-Mass Spectrometry," Clinical Chemistry, Vol. 40, No. 1, 1994, pp. 71-73.

[3] Y. Sano, A. Sakamoto, Y. Oi and R. Ogawa, "Anaesthesia and Circulating Blood Volume," European Journal of Anaesthesiology, Vol. 22, No. 4, 2005, pp. 258-262. http://dx.doi.org/10.1017/S0265021505000438

[4] H. L. Webster, "Colloid Osmotic Pressure: Theoretical Aspects and Background," Clinics in Perinatology, Vol. 9, No 3, 1982, pp. 505-521.

[5] T. H. Allen, M. T. Peng, K. P. Chen, T. F. Huang, C. Chang and H. S. Fang, "Prediction of Blood Volume and Adiposity in Man from Body Weight and Cube of Height," Metabolism, Vol. 5, No. 3, 1956, pp. 328-345.

[6] R. G. Hahn, "Blood Volume at the Onset of Hypotentin during TURP Performed under Epidural Anaesthesia," European Journal of Anaesthesiology, Vol. 10, No 3, 1993, pp. 219-225.

[7] D. L. Bourke and T. C. Smith, "Estimating Allowable Hemodilution," Anesthesiology, Vol. 41, No. 6, 1974, pp. 609-611.

http://dx.doi.org/10.1097/00000542-197412000-00015

[8] M. Rehm, V. Orth, U. Kreimeier, M. Thiel, M. Haller, H. Brechtelsbauer and U. Finsterer, "Changes in Intravascular Volume during Acute Normovolemic Hemodilution and Intraoperative Retransfusion in Patients with Radical Hysterectomy," Anesthesiology, Vol. 92, No. 3, 2000, pp.
657-664.

http://dx.doi.org/10.1097/00000542-200003000-00008

[9] A. R. Pries, T. W. Sevomb and P. Gaehtgens, "The Endothelial Surface Layer," Pflügers Archiv: European Journal of Physiology, Vol. 440, No. 5, 2000, pp. 653-666.

[10] A. R. Pries, A. Fritzsche, K. Ley and P. Gaehtgens, "Redistribution of Red Blood Cell Flow in Microcirculatory Networks by Hemodilution," Circulation Research, Vol. 70, No. 6, 1992, pp. 1113-1121. http://dx.doi.org/10.1161/01.RES.70.6.1113

[11] J. D. Oliver III, S. Anderson, J. L. Try, B. M. Brenner and W. H. Deen, "Determination of Glomerular Size-Selectivity in the Normal Rat with Ficoll," Journal of the American Society of Nephrology, Vol. 3, No. 2, 1992, pp. 214228.

[12] J. F. Danielli, "Capillary Permeability and Edema in the Perfused Frog," The Journal of Physiology, Vol. 98, No. 1, 1940, pp. 109-129.

[13] R. Chambers and B. W. Zweifach, "Intracellular Cement and Capillary Permeability," Physiological Reviews, Vol. 27, No. 3, 1947, pp. 436-463.

[14] M. Rehm, S. Zahler, M. Lötsch, U. Welsch, P. Conzen, M. Jacob and B. F. Becker, "Endo Thelial Glycocalyx as an Additional Barrier Determining Extravasation of 6\% Hydroxyethyl Starch or 5\% Albumin Solutions in the Coronary Vascular Bed," Anesthesiology, Vol. 100, No. 5, 2004, pp. 1211-1223. http://dx.doi.org/10.1097/00000542-200405000-00025

[15] M. Jacob, D. Bruegger, M. Rehm, M Stoeckelhuber, U. Welsch, P. Conzen and B. F. Becker, "The Endothelial Glycocalyx Affords Compatibility of Starling's Principle and High Cardiac Interstitial Albumin Levels," Cardiovascular Research, Vol. 73, No. 3, 2007, pp. 575-586. http://dx.doi.org/10.1016/j.cardiores.2006.11.021

[16] C. R. Bell, P. J. Murdock, K. J. Pasi and R. J. Morgan, "Thrombotic Risk Factors Associated with Transurethral Prostatectomy," BJU International, Vol. 83, No. 9, 1999, pp. 984-989. 